\title{
Hábitos alimentares e ocorrência de alterações no metabolismo lipídico em universitários residentes e não residentes com familiares
}

\author{
Food habits and occurrence of changes in lipid metabolism \\ in resident and non-resident university with family
}

Recebido em: 19/07/2018 Aceito em: $\quad 16 / 10 / 2018$
Vanderlan Nogueira HOLANDA ${ }^{1}$; Dayane Correia GOMES ${ }^{2}$; Elton Marlon de Araújo LIMA ${ }^{3}$; Sherida Brito de FREITAS ${ }^{4}$; Wislayane Gomes MILFONT ${ }^{5}$;

Vivianne Cortez Sombra VANDESMET ${ }^{4}$ ${ }^{1}$ Laboratório de Lipídios, Departamento de Bioquímica, Universidade Federal de Pernambuco - UFPE. Avenida Prof. Moraes Rego, s/n, Cidade Universitária, CEP 50670-420. Recife, PE, Brasil. ${ }^{2}$ Laboratório de Biologia Molecular, Departamento de Bioquímica, Universidade Federal de Pernambuco - UFPE. Avenida Prof. Moraes Rego, s/n, Cidade Universitária, CEP 50670-420. Recife, PE, Brasil. ${ }^{3}$ Laboratório de Polímeros Não-convencionais, Departamento de Física, Universidade Federal de Pernambuco - UFPE. Av. Jorn. Aníbal Fernandes, s/n, Cidade Universitária, CEP 50740-540. Recife, PE, Brasil. ${ }^{4}$ Centro Universitário Doutor Leão Sampaio UNILEÃO. Av. Leão Sampaio km 3, Lagoa Seca, CEP 63040-005. Juazeiro do Norte, Ceará, Brasil. ${ }^{5}$ Universidade Regional do Cariri - URCA. Rua Coronel Antônio Luíz, 1161, Pimenta, CEP 63105-010. Crato, Ceará, Brasil. E-mail: vanderlan.nogueira@gmail.com

\section{ABSTRACT}

Dyslipidemia consists of a change related to lipid metabolism and is among the leading causes of cardiovascular diseases in the world, affecting different populations, especially university students. Admission to the university is a stage full of biopsychosocial changes and among them, leaving the parents' home is directly related to changes in eating habits and consequently changes in lipid metabolism. In this study, the eating habits and the lipid profile of a population of 86 university students living with or without their parents, from a higher education institution in Juazeiro do Norte, Ceará, Brazil, were evaluated. Of the 86 participants in the study; $55.8 \%$ were female, and $44.2 \%$ were male; $95.4 \%$ of university students presented poor or irregular eating habits, and only $4.6 \%$ presented healthy eating. The most common changes in the lipid profile were hypertriglyceridemia and decreased HDL-c. From the results of the present research, it is possible to conclude that university students living with their parents are more likely to adopt poor eating habits and develop dyslipidemias. The more active participation of higher education Institutions in campaigns that promote more information about the advantages of adopting a healthier lifestyle would constitute an important preventive action for university students.

Keywords: dyslipidemias; feeding behavior; students. 
A dislipidemia consiste numa alteração relacionada ao metabolismo lipídico e está entre as principais causas de doenças cardiovasculares no mundo, acometendo diferentes populações, sobretudo estudantes universitários. O ingresso na universidade é uma etapa repleta de mudanças biopsicossociais e dentre elas, a saída da casa dos pais está diretamente relacionada a modificações nos hábitos alimentares e consequentemente alterações no metabolismo lipídico. Neste estudo, foram avaliados os hábitos alimentares e determinado o perfil lipídico de uma população de 86 estudantes universitários que viviam ou não com os pais, oriundos de uma instituição de ensino superior de Juazeiro do Norte, Ceará, Brasil. Dos 86 participantes do estudo, $55,8 \%$ eram do sexo feminino e 44,2\% do masculino; $95,4 \%$ dos universitários apresentaram hábitos alimentares inadequados ou irregulares ao estilo de vida saudável; e apenas $4,6 \%$ apresentaram a alimentação saudável. As alterações mais prevalentes no perfil lipídico foram hipertrigliceridemia e diminuição do HDL-c. A partir dos resultados da presente pesquisa foi possível concluir que os estudantes universitários que vivem com os pais apresentam uma maior probabilidade de adoção de maus hábitos alimentares e desenvolvimento de dislipidemias. A participação mais ativa de Instituições de Ensino Superior em campanhas que promovam maior informação sobre as vantagens da adoção de um estilo de vida mais saudável se constituiria de uma importante ação preventiva para os universitários.

Palavras-chave: dislipidemia; comportamento alimentar; estudantes

\section{INTRODUÇÃO}

A dislipidemia consiste numa alteração relacionada ao metabolismo lipídico, podendo ser caracterizada pelo aumento do colesterol plasmático e suas frações: lipoproteína de baixa densidade (LDL-c) e triglicerídeos (TG) ou diminuição da lipoproteína de alta densidade (HDL-c) (1). Esta desordem metabólica está entre as principais causas de doenças cardiovasculares no mundo e acomete diferentes populações, sobretudo estudantes universitários, tornando-se um problema de saúde pública pela associação com diferentes morbidades (2).

A dieta inadequada associada ao sedentarismo contribui com o aumento da ocorrência de doenças crônicas não transmissíveis (DCNT), especialmente doenças cardiovasculares. Em 2013, a doença cardiovascular aterosclerótica correspondeu quase que um terço do total de óbitos em todo o mundo (3) e atualmente no Brasil, as DCNT correspondem a $72 \%$ das causas de morte, com maior incidência na população de baixa renda (4). O surgimento da aterosclerose tem acometido cada vez mais adultos jovens e gerado altos gastos para a saúde pública. Os medicamentos para tratamento de dislipidemias e agentes modificadores de lipídios correspondem, somados, a $27 \%$ dos dispêndios executados pelo Ministério da Saúde no serviço de Farmácia Popular no período correspondente de 2006 a 2014 (5).

Dentre os grupos mais vulneráveis ao desenvolvimento de dislipidemias estão os estudantes universitários, população majoritariamente composta por adultos jovens (6). O risco de doença coronariana, excesso de peso e dislipidemia em adultos jovens aumenta de 2 a $4 \%$ ao ano (7), quando considerado o contexto universitário; tem sido observada a prevalência de sobrepeso, diabetes mellitus, doenças cardiovasculares e dislipidemia, devido ao estabelecimento de hábitos alimentares desapropriados e ao alto nível de sedentarismo $(8,9)$.

Estudos mostram que a exposição à vida universitária resulta no aumento de colesterol e isso pode estar associado às mudanças que muitos estudantes fazem ao ingressarem nas diversas instituições de ensino superior $(10,11)$. O ingresso na universidade é uma etapa repleta de mudanças biopsicossociais e, dentre elas, a saída da casa dos pais está diretamente relacionada a modificações nos hábitos alimentares, caracterizados principalmente pela busca de alternativas práticas e rápidas, resultando no consumo de alimentos industrializados, mais calóricos e com menor qualidade nutricional (12). 
A avaliação do perfil lipídico e dos hábitos alimentares de universitários que moram com ou sem a presença dos pais pode ser uma alternativa importante para minimizar os riscos de dislipidemias e doenças correlacionadas neste grupo. Logo, o presente estudo avaliou os hábitos alimentares e investigou a ocorrência de alterações no perfil lipídico em um grupo de estudantes universitários de uma Instituição de Ensino Superior da cidade de Juazeiro do Norte, Região do Cariri, CE.

\section{MÉTODOS}

Caracterização da População Estudada. Participaram da pesquisa 86 estudantes universitários matriculados nos cursos de graduação da área da Saúde de uma instituição de ensino de Juazeiro do Norte, CE, Brasil. Foram inclusos na pesquisa 44 universitários que, no momento da coleta de dados, residiam com os pais (ERP) e 42 que não residiam com os pais (ENRP). Não participaram os estudantes que não tinham integralizado pelo menos um semestre letivo até a coleta de dados. A seleção dos participantes ocorreu de forma aleatória a partir da manifestação voluntária a participar deste estudo. A presente pesquisa ocorreu em duas etapas (Figura 1).

Avaliação dos Hábitos Alimentares - Etapa I. $\mathrm{O}$ estudo foi dividido em duas etapas: a primeira etapa foi constituída pela aplicação de um questionário composto por 18 perguntas correspondentes ao consumo de alimentos, adaptado do Manual de Hábitos Alimentares proposto pelo Ministério da Saúde (13).

No questionário foram avaliadas as seguintes variáveis: sexo, idade, curso, semestre, moradia, prática de exercícios físicos, quantidade de consumo de frutas, de verduras ou legumes, de cereais, arroz, farinha ou macarrão, de carnes, frituras, tipos de gorduras utilizadas no preparo de refeições, consumo de sal e realização do almoço como principal refeição do dia.

As informações foram registradas numa planilha e cada participante foi identificado por um código numérico seguido de suas iniciais. Após a entrega do questionário o participante foi orientado a realizar de $10 \mathrm{~h}$ a $12 \mathrm{~h}$ de jejum, não ingerir álcool e manter alimentação habitual e não realizar atividade física no dia que antecedeu a coleta de amostra sanguínea.

\section{Determinação do Perfil Lipídico - Etapa II.} A segunda etapa consistiu na determinação do perfil lipídico. Para isso, os participantes foram orientados sobre o preparo para a realização do exame e, posteriormente, foram coletadas as amostras de sangue sob supervisão de profissional habilitado em Análises Clínicas.

As amostras foram colhidas e processadas, acondicionadas em tubos estéreis sem adição de anticoagulantes e identificadas com o mesmo código numérico gerado no momento da aplicação do questionário. Em seguida, foi realizada a dosagem da Lipoproteína de Alta Densidade (HDL-c), Triglicerídeos (TG) e Colesterol Total (CT), por meio do método enzimático-colorimétrico no analisador bioquímico Bayer RA-XT®. A dosagem dessas lipoproteínas aconteceu com a utilização dos Kits Colesterol Liquiform ${ }^{\circledR}$, Triglicerides Liquiform ${ }^{\circledR}$ e HDL LE, em cada uma das análises foram seguidas as recomendações do fabricante.

A determinação das frações da Lipoproteína de Muito Baixa Densidade (VLDL) e Lipoproteína de Baixa Densidade (LDL) foi feita a partir da aplicação de fórmula de Friedwald: $\mathrm{LDL}=$ Colesterol Total - (HDL + Triglicerídeos/5) (14). Após os dados serem agrupados em tabelas do Microsoft Excel®, foram determinados os índices de Castelli. 
Primeira Etapa Avaliação dos Hábitos Alimentares
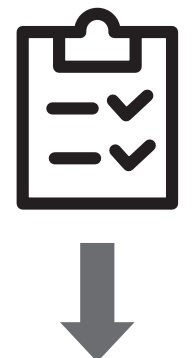

18 Questões
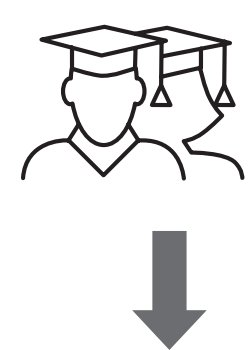

86 Universitários

Caracterização dos participantes 44 ERP e 42 ENRP

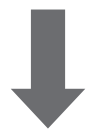

Orientações para realização da segunda etapa: dosagem do perfil lipídico

Segunda Etapa

Determinação do Perfil Lipídico
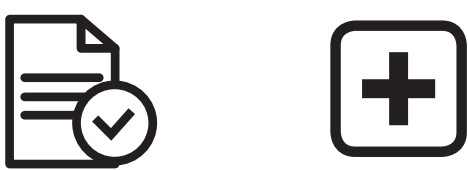

Assinatura do TCLE/TCLPE e verificação do seguimento das recomendações pré-coleta
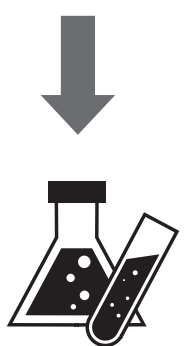

Determinação Laboratorial do Perfil Lipídico

Figura 1. Métodos e Procedimentos para Avaliação dos Hábitos Alimentares e Determinação do Perfil Lipídico de estudantes universitários de Juazeiro do Norte, Ceará, Brasil

Análise Estatística. Foi elaborado um banco de dados no programa estatístico SPSS ${ }^{\circledR}$, versão 20, a partir do qual foram realizados procedimentos analíticos voltados para apresentação descritiva dos resultados e testagem das hipóteses de interesse desta pesquisa. As variáveis quantitativas foram testadas em relação a sua normalidade, apresentando adequação para validar o uso dos testes paramétricos.

Foram empregados os seguintes testes: teste $\mathrm{t}$ de Student para amostras independentes a partir das variáveis bioquímicas avaliadas em relação às categorias de sexo, atividade física, semestre, residência e comportamento alimentar. Em relação aos cruzamentos entre as variáveis categóricas, empregou-se o teste de Pearson Chi-Square. Por fim, foi utilizado o teste de ANOVA (Análise de Variância Oneway) visando identificar possíveis diferenças entre os valores bioquímicos em função dos cursos pesquisados. Em todos os testes foi adotado um $\mathrm{p} \leq 0,05$ para assumir significância estatística. Os resultados foram apresentados na forma de tabelas visando uma maior legibilidade.

Aspectos Éticos da Pesquisa. Somente participaram desta pesquisa os indivíduos que assinaram o Termo de Consentimento Livre Esclarecido (TCLE) e Termo de Consentimento Livre Pós-Esclarecido (TCLPE). A presente pesquisa foi realizada de acordo com as normas éticas estabelecida pela legislação brasileira e obteve parecer favorável à sua execução pelo Comitê de Ética em Pesquisa, com autorização registrada no CAAE: 47773815.0.0000.5048.

\section{RESULTADOS E DISCUSSÃO}

Dos 86 participantes do estudo, $55,8 \%$ foram do sexo feminino e $44,2 \%$ do masculino. Participaram da pesquisa 33 alunos da Biomedicina, 11 alunos da Fisioterapia, 13 alunos da Psicologia, 12 alunos da Enfermagem e 17 alunos da Odontologia (Tabela 1). 
Tabela 1. Distribuição dos estudantes universitários, de Juazeiro do Norte, Ceará, Brasil, sujeitos da pesquisa, segundo sexo, curso e idade.

\begin{tabular}{|c|c|c|c|c|c|c|}
\hline \multirow{2}{*}{ Curso } & \multicolumn{3}{|c|}{ Masculino } & \multicolumn{3}{|c|}{ Feminino } \\
\hline & $\mathbf{n}$ & $\%$ & Idade (anos)* & $n$ & $\%$ & Idade (anos)* \\
\hline Biomedicina & 13 & 39,4 & $20,15 \pm 0,80$ & 20 & 60,6 & $19,70 \pm 0,80$ \\
\hline Psicologia & 08 & 61,5 & $20,25 \pm 1,66$ & 05 & 38,5 & $20,20 \pm 1,30$ \\
\hline Enfermagem & 03 & 25 & $20,66 \pm 1,15$ & 09 & 75,0 & $21,22 \pm 3,03$ \\
\hline Odontologia & 08 & 47,0 & $20,50 \pm 1,69$ & 09 & 53,0 & $19,88 \pm 1,36$ \\
\hline Fisioterapia & 06 & 54,5 & $20,33 \pm 1,03$ & 05 & 45,5 & $19,60 \pm 0,89$ \\
\hline
\end{tabular}

Valores de idade expressos em média \pm desvio-padrão.

Em um estudo realizado com universitários de uma instituição pública do Piauí (11), a faixa etária dos participantes foi de 18 a 25 anos, com média de 22,6 anos, dados semelhantes aos encontrados na presente pesquisa. A média de idade dos participantes deste estudo pode estar relacionada ao fato do grupo de universitários ser composto majoritariamente por adultos jovens. É importante destacar que a ocorrência de doenças crônicas relacionadas ao metabolismo lipídico nessa faixa etária é subestimada, mesmo ocorrendo alta prevalência de fatores de risco, dificultando a notificação dessas enfermidades nestas populações (7).

Em relação à avaliação dos hábitos alimentares, 95,4\% dos universitários apresentaram hábitos alimentares inadequados ou regulares ao estilo de vida saudável e apenas 4,6\% apresentaram a alimentação saudável, conforme classificação do Guia Alimentar do Ministério da Saúde (13). Os resultados da presente pesquisa estão de acordo com os achados da Pesquisa Nacional de Saúde (15), que destacou o preocupante alto índice de consumo de alimentos não saudáveis no Brasil. (16), em seu estudo sobre prevalência de comportamentos de risco em adulto jovem e universitário destacam os hábitos alimentares pouco saudáveis como fator de risco na população estudada, cuja prevalência de excesso de peso era de $25 \%$. O baixo percentual de hábitos alimentares saudáveis na população deste estudo pode ser devido ao ingresso no ensino superior estar relacionado com a diminuição de tempo para alimentação, onde há a troca de refeições por fast-food (17).

A inadequação de hábitos alimentares vem sendo citada em outros estudos sobre hábitos ali- mentares de universitários no decorrer desta década (18). O consumo insuficiente de frutas e hortaliças é prevalente numa população de estudantes universitários de uma Instituição de Ensino Superior (IES) pública da região sul do Estado da Bahia, promovendo assim o estabelecimento de uma alimentação irregular. A má alimentação dos participantes deste estudo pode estar associada aos valores culturais locais, uma vez que esse fator tende a ser mais marcante na população adulta e com maior escolaridade (19).

Diversos estudos epidemiológicos têm ressaltado evidências sobre a importância da dieta como um dos principais fatores de risco para doenças cardiovasculares. Numerosos alimentos e nutrientes têm sido relacionados ao estabelecimento de doenças crônicas não transmissíveis em populações distintas, sobrelevando o consumo excessivo de colesterol e gorduras saturadas, alterando de maneira expressiva o perfil lipídico (20).

Após a associação das categorias ERP e ENRP com os hábitos alimentares, por meio do teste Pearson Chi-Square, os indivíduos do grupo ERP apresentaram diferenças estatisticamente significantes, quando comparados aos do grupo ENRP (Tabela 2).

Tais achados podem estar relacionados aos valores culturais e influência do seio familiar, tendo em vista a possibilidade de existir um comportamento alimentar inadequado pré-existente entre familiares, onde eles próprios não se sentem confortáveis quando são questionados sobre os próprios hábitos alimentares (21). Estes resultados corroboram com os achados por outros pesquisa- 
dores, que ao realizarem um estudo sobre a relação do ambiente alimentar e convívio familiar, destacaram que conviver com a família não representa proteção à vulnerabilidade familiar (22). Porém, a existência de um fator inerente à relação familiar é destacada em depoimentos coletados por Alves e Boog (2007), nos quais estudantes que saíram das residências paternas para viverem em moradias universitárias destacam que o sentido afetivo altera muito a alimentação, não sendo a mesma situação comer com a família e comer em restaurantes universitários (23).

Tabela 2. Hábitos Alimentares de estudantes universitários, de Juazeiro do Norte, Ceará, Brasil, segundo a condição de Convívio Familiar

\begin{tabular}{|c|c|c|c|c|}
\hline \multirow{2}{*}{ Hábitos Alimentares } & \multicolumn{2}{|c|}{ ERP } & \multicolumn{2}{|c|}{ ENRP } \\
\cline { 2 - 5 } & $\mathbf{n}$ & $\%$ & 39 & $\%$ \\
\hline Adequado & 27 & $61,4 \%^{* *}$ & 3 & $92,9 \% * *$ \\
\hline Inadequado & 17 & $38,6 \% * *$ & $7,1 \% * *$ \\
\hline
\end{tabular}

**Valores estatisticamente significantes $(p \leq 0,05)$. ERP: residiam com os pais; ENRP: não residiam com os pais

Com relação à prática de atividade física, $72 \%$ dos estudantes não realizavam, no mínimo $30 \mathrm{mi}-$ nutos de atividade física diária (Figura 2). Petribú e cols. (2009), também encontraram em seus estudos relevantes taxas de sedentarismo $(41,7 \%)$, excesso de peso $(40,8 \%)$ e obesidade $(20,2 \%)$ em estudantes universitários (24). Esse alto sedentarismo também foi encontrado pela pesquisa realizada por Marcondelli e cols. (2008), onde foram analisados os níveis de atividade física e hábitos alimentares de universitários da área da Saúde, concluindo que $65,5 \%$ da população estudada eram sedentários, com destaque aos alunos dos cursos de Enfermagem, Medicina e Odontologia (8).

$\mathrm{O}$ alto índice de sedentarismo também foi observado quanto ao avanço dos universitários no curso de graduação. À medida que o estudante segue progressivamente no curso, a taxa de sedentarismo é mais prevalente, mesmo não havendo diferenças significativas entre os primeiros e últimos anos da graduação (24).

Dentre os cursos avaliados, os estudantes que, independente de residirem ou não com os pais, que mais afirmaram praticar atividade física foram do curso de Fisioterapia, dos quais $72,7 \%$ afirmaram realizar pelo menos 30 minutos de atividade física diária. Isso se deve ao fato de que a matriz curricular do curso oferece disciplinas práticas voltadas para o exercício aplicado à reabilitação, como por exemplo, a Terapia Manual onde o acadêmico utiliza técnicas corporais, durante as aulas práticas que exigem condicionamento físico para se obter um melhor desempenho.

Assim como os estudantes de Fisioterapia, os acadêmicos do curso de Educação Física da Universidade de Brasília também apresentaram menores índices de sedentarismo, corroborando assim com a ideia de que as atividades curriculares inerentes a estes cursos oportunizam o estudante a realizar, em alguns momentos, o mínimo de atividade física por dia estabelecida pela Organização Mundial de Saúde (8). Ainda que os estudantes destas áreas realizem o mínimo de atividade física durante os cursos, o contexto universitário contribui sobremaneira para o sedentarismo, como foi observado em estudo em que à medida que o acadêmico de Educação Física avança no curso, há a diminuição da prática de exercícios físicos regulares (26). 


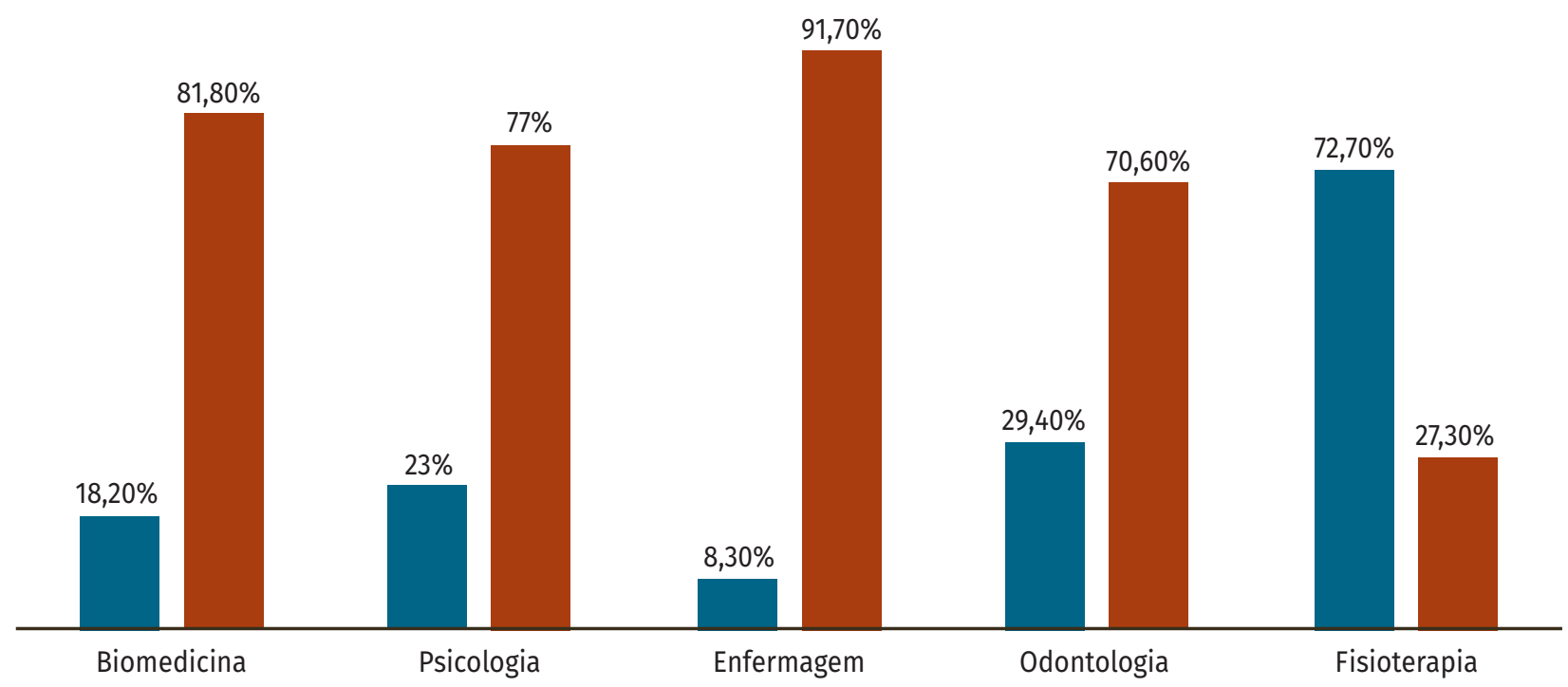

Figura 2. Percentual de estudantes universitários, de Juazeiro do Norte, Ceará, Brasil, que praticavam atividade física, por Curso de Graduação

Outro fator importante é o tempo que tais indivíduos necessitam para dedicar à prática de atividades físicas regulares, pois quanto mais as atividades curriculares aumentam, mais atarefados estes se encontram. Em uma pesquisa realizada na Universidade Federal de Pelotas, Rio Grande do Sul, foram analisados 485 universitários de 17 cursos que compuseram quatro áreas: Ciências Biológi- cas, Ciências Exatas, Ciências Humanas, Ciências Agrárias. O estudo mostrou que $86 \%$ dos acadêmicos relataram gostar de prática de atividades físicas, mas $60 \%$ afirmaram não ter tempo livre para praticá-las (27). Para a associação entre hábitos alimentares e prática de atividade física não houve diferenças estatísticas relevantes após aplicação do teste de Qui-quadrado (Tabela 3).

Tabela 3. Associação entre moradia universitária e prática de atividade física regular entre estudantes universitários, de Juazeiro do Norte, Ceará, Brasil

\begin{tabular}{|c|c|c|c|c|c|c|}
\hline \multirow{2}{*}{ Hábitos alimentar } & \multicolumn{2}{|c|}{ ERP } & \multicolumn{2}{|c|}{ ENRP } & & \\
\hline & $n$ & $\%$ & $n$ & $\%$ & $x^{2}$ & $\mathbf{p}$ \\
\hline Realiza AF & 9 & $20,5 \%$ & 14 & $33,3 \%$ & 1,819 & 0,177 \\
\hline Não realiza AF & 35 & $79,5 \%$ & 28 & $66,7 \%$ & & \\
\hline
\end{tabular}

AF: Atividade Física; ERP: Estudantes que Residem com os Pais; ENRP: Estudantes que Não Residem com os Pais

Quando analisado o perfil lipídico da população deste estudo, foi observado que os níveis médios de triglicerídeos se apresentaram maiores nos estudantes com hábitos alimentares inadequados ou regulares, sendo estatisticamente significante (Tabela 4), quando comparados às outras frações do perfil lipídico. Diversos estudos têm apontado a alta prevalência de hipertrigliceridemia em estu- dantes universitários e isso se deve ao fato que essa fração do perfil lipídico é modulada pelos hábitos alimentares (9). Similarmente, no estudo feito por Correa-Bautista e cols. (2017), onde foi determinando o perfil lipídico de universitários com faixa etária entre 18 e 35 anos, também foi encontrada prevalência de hipertrigliceridemia e associações com outras frações do lipidograma (28). 
Tabela 4. Associação entre Hábitos Alimentares e Perfil Lipídico em estudantes universitários, de Juazeiro do Norte, Ceará, Brasil

\begin{tabular}{|l|c|c|c|c|}
\hline & ADEQUADO & DP & INADEQUADO & DP \\
\hline TRIGLICERÍDEOS & $107,5^{* *}$ & 43,8 & $156,9^{* *}$ & 74,7 \\
\hline CT & 163,7 & 30,6 & 177,6 & 41,4 \\
\hline HDL-C & 43,7 & 8,5 & 46,9 & 8,6 \\
\hline LDL-C & 98,5 & 30,5 & 99,4 & 40,6 \\
\hline CASTELLI I & 3,9 & 1,0 & 3,9 & 1,0 \\
\hline CASTELLI II & 2,4 & 0,9 & 2,2 & 1,0 \\
\hline
\end{tabular}

DP: Desvio Padrão; CT: Colesterol Total; HDL-c: Lipoproteína de Alta Densidade; LDL-c: Lipoproteína de Baixa Densidade. Valores estatisticamente significantes $(p \leq 0,01)$

Segundo a atualização da Diretriz Brasileira de Dislipidemia e Prevenção da Aterosclerose 2017, níveis elevados de triglicerídeos estão associados a baixos níveis de HDL-c, fato este observado na presente pesquisa. A Hipertrigliceridemia isolada se constitui de uma dislipidemia grave, podendo haver acometimento pancreático a longo prazo, necessitando de intervenções de restrição alimentar e farmacológica (30).
A fração do perfil lipídico que apresentou um maior índice de variação foram os níveis médios de HDL-c, onde 30,2\% dos universitários apresentaram a HDL-c abaixo dos valores de referência, $64 \%$ estavam nos limites da normalidade e apenas $5,8 \%$ apresentaram valores desejáveis. $\mathrm{O}$ sexo feminino apresentou níveis estatisticamente mais positivos quando comparados aos indivíduos do sexo masculino (Tabela 5).

Tabela 5. Perfil Lipídico em estudantes universitários, de Juazeiro do Norte, Ceará, Brasil, de acordo com o gênero

\begin{tabular}{|l|c|c|c|c|}
\hline & MASCULINO & DP & FEMININO & DP \\
\hline TRIGLICERIIDEOS & 120,8 & 59,0 & 117,5 & 54,5 \\
\hline CT & 169,7 & 30,4 & 164,8 & 36,3 \\
\hline HDL-C & $40,5^{* *}$ & 6,6 & $47,6^{* *}$ & 8,7 \\
\hline LDL-C & 105,0 & 28,2 & 93,6 & 35,6 \\
\hline CASTELLII & $4,3^{* *}$ & 0,9 & $3,6^{* *}$ & 0,9 \\
\hline CASTELLI II & $2,7^{* *}$ & 0,9 & $2,0^{* *}$ & 0,9 \\
\hline
\end{tabular}

DP: Desvio Padrão; CT: Colesterol Total; HDL-c: Lipoproteína de Alta Densidade; LDL-c: Lipoproteína de Baixa Densidade. Valores estatisticamente significantes $(p \leq 0,01)$

Floody e cols (2015) também encontraram níveis baixos de HDL-c ao determinarem o perfil lipídico dos estudantes da Universidad Santo Tomás, Temuco, Chile (31). A ocorrência simultânea do aumento de triglicerídeos e diminuição de HDL-c, observada na presente pesquisa, também foi encontrada num estudo realizado com graduandos de universidades públicas e privadas de Bogotá e Cali, Colômbia (28). Carvalho e cols. (2015), destacaram que a diminuição do HDL-c constitui um importante parâmetro que condiciona a ocorrência de doenças do aparelho circulatório e justificaram esse achado ao fato da maioria dos universitários não realizarem atividade física regular.

Estando de acordo com os achados desta pesquisa, Moreira e cols; (2011), determinaram os fatores de risco coronarianos em estudantes de uma universidade privada e notaram que os indivíduos do sexo masculino apresentaram maior risco quando comparados as mulheres (33). No presente estudo, o risco coronariano, determinado pelos Índices de Castelli I e II também apresentou 
diferenças estatísticas significantes com índices mais elevados nos indivíduos de sexo masculino. Resultados semelhantes foram encontrados num estudo feito com estudantes da Universidad de Guadalajara, México, que mostrou que a população masculina apresentou 3 vezes mais alterações nos níveis de triglicerídeos, quando comparados as mulheres (34).

Esses resultados podem estar associados à variabilidade biológica determinada pelos hormônios estrogênio e progesterona, inerentes ao sexo feminino, por conferirem maior vasodilatação re- tardando assim o desenvolvimento aterosclerótico nas mulheres (35).

Os estudantes que estavam nos semestres mais avançados do curso apresentaram índices de Castelli I significantemente melhores do que os estudantes dos semestres iniciais (Tabela 6). Para melhor representá-los, os universitários foram organizados em dois grupos: os que estavam matriculados nos respectivos cursos de ensino superior até 2 anos e os que estavam a partir de 2 anos. A média de duração dos cursos em que os estudantes estavam matriculados era de 4,8 anos.

Tabela 6. Associação entre os Índices de Castelli com o Período de Graduação entre estudantes universitários, de Juazeiro do Norte, Ceará, Brasil

\begin{tabular}{|l|c|c|c|c|}
\hline & ATÉ 2 ANOS & DP & A PARTIR DE 2 ANOS & DP \\
\hline CASTELLI I & $4,2^{*}$ & 1,1 & $3,7^{*}$ & 0,9 \\
\hline CASTELLI II & 2,5 & 1,0 & 2,2 & 0,9 \\
\hline
\end{tabular}

Valores estatisticamente significantes $(p \leq 0,05)$

Segundo Pereira e cols. (2009), tal achado pode ser explicado pelo fato de que um maior nível de escolaridade está associado a um menor risco cardiovascular em indivíduos de ambos os sexos, ressaltando os resultados da presente pesquisa (36). O ingresso na vida universitária está, muitas vezes, associado a manifestações de estresse que podem desencadear maus hábitos alimentares e estabelecimento de sedentarismo. Nesse contexto, Rovida e cols. (2015), também encontraram fatores associados ao estilo de vida não saudável numa população de estudantes ingressantes de um curso de Odontologia (37). No Brasil, indivíduos com educação universitária apresentam menor probabilidade de doença cardiovascular no futuro (38). Para as associações de perfil lipídico e cursos de graduação, não houve diferenças estatísticas significantes.

\section{CONCLUSÃO}

A partir dos resultados da presente pesquisa é possível concluir que os estudantes universitários que vivem com os pais apresentam uma maior probabilidade de adoção de maus hábitos alimentares e desenvolvimento de dislipidemias. Durante a in- fância e a adolescência o indivíduo realiza atividade física de forma mais ativa, seja no âmbito social ou escolar. Quando se encerra o ensino médio, o jovem ou adulto ingressa no ensino superior tendo muitas vezes que conciliar trabalho e estudo, não dispondo de tempo para a prática de atividades físicas. Os estudos que visam à caracterização do perfil lipídico e risco de uma determinada população desenvolver acometimentos cardiovasculares se constitui da primeira etapa para elaboração e execução de ações voltadas para promoção e prevenção da saúde aplicadas à população estudada. A participação mais ativa de Instituições de Ensino Superior por meio da promoção de campanhas que divulguem maior informação sobre as vantagens da adoção de um estilo de vida mais saudável se constituiria de uma importante ação preventiva para os universitários.

\section{AGRADECIMENTOS}

Os autores agradecem ao Centro Universitário Leão Sampaio (UNILEÃO) pela infraestrutura disponibilizada, em especial o apoio da Coordenação de Pesquisa e Extensão, essencial para realização desta pesquisa. 
1. Nieto CIR, Pérez JDM, Freire LM, Morales KRDP, Vicente ERC. Prevalencia de síndrome metabólico y factores de riesgo asociados en jóvenes universitarios ecuatorianos. Nut Hospitalaria. 2015; 31(4):1581. DOI: 10.3305/nh.2015.31.4.8371.

2. Santos RD, Bensenor IM, Pereira AC, Lotufo PA. Dyslipidemia according to gender and race: The Brazilian Longitudinal Study of Adult Health (ELSA-Brasil). J Clin Lipidol. 2016; 10(6): 1368. DOI: 10.1016/j. jacl.2016.08.008.

3. GDB. Global Burden of Disease. Global, regional, and national age-sex specifi c all cause and cause-specifi c mortality for 240 causes of death, 1990-2013: a systematic analysis for the Global Burden of Disease Study 2013. The Lancet 2014; 9963 (385): 117-171.

4. Rocha RM, Martins WA. Manual de Prevenção Cardiovascular. 1 ed. São Paulo: Planmark, 2017.

5. Silva RM, Caetano R. Gastos com pagamentos no Programa Aqui Tem Farmácia Popular: evolução entre 2006-2014. Rev Saúde Coletiva. 2018; 28(1):1-30. DOI: 10.1590/S0103-73312018280105.

6. Torquato SC, Silva FS, Rodrigues AR, Pimenta MVT, Moura JRA, Silva ARV. Sedentary lifestyle and metabolic disorders among college. Rev Enf UFPI. 2016;5(2):1621.

7. Arts J, Fernandez ML, Lofgren IE. Coronary Heart Disease Risk Factors in College Students. Adv Nutrition. 2014; 5(2): 177-187. DOI: 10.3945/an.113.005447.

8. Marcondelli P, Costa THM, Schimitz BAS. Nível de Atividade Física e Hábitos Alimentares de Universitários do $3^{\circ}$ ao $5^{\circ}$ semestres da área da saúde. Rev Nutrição. 2008; 21(1): 39-47. DOI: 10.1590/S1415-52732008000100005.

9. AlMajed HT, AlAttar AT, Sadek AA, AlMuaili AA, AlMutairi OA, Shaghouli AS, AlTorah WA. Prevalence of dyslipidemia and obesity among college students in Kuwait. Alexandria J Medicine. 2011; 47(1): 67-71. DOI: 10.1016/j.ajme.2010.12.003.

10. Freitas RWJF, Araújo MFM, Lima ACS, Pereira DCR, Alencar ANPG, Damasceno MMC. Análise do perfil lipídico de uma população de estudantes universitários. Rev Lat-Amer Enf. 2013; 21(5): 1-8.

11. Moura JRA, Guimarães MR, Teixeira SEXM, Carvalho EL, Machado ALG, Silva ARV. Lipid profile of university and association with cardiovascular risk. Rev Enf UFPI. 2017; 6(1):40-46.

12. Santos AKGV, Reis CC, Chaud DMA, Morimoto JM. Qualidade de vida e alimentação de estudantes universitários que moram na região central de São Paulo sem a presença dos pais ou responsáveis. Rev Simbio-Logias. 2014; 7(10):76-99.
13. BRASIL. Ministério da Saúde. Guia alimentar: como ter uma alimentação saudável. 2015.

14. Friedewald WT, Robert IL, Fredrickson DS. Estimation of the Concentration of Low-Density Lipoprotein Cholesterol in Plasma, Without Use of the Preparative Ultracentrifuge. Clinical Chem. 1972; 18(6): 499-502.

15. BRASIL. Ministério da Saúde. Pesquisa Nacional de Saúde. 2015.

16. Faria YO, Gandolfi L, Moura LBA. Prevalência de comportamentos de risco em adulto jovem e universitário. Acta Paulista Enf. 2014; 27(6): 591-5. DOI: 10.1590/1982 - 0194201400096.

17. Busato MA, Predrolo C, Gallina LS, Rosa L. Ambiente e alimentação saudável: percepções e práticas de estudantes universitários. Semina;2015; 36(2): 75-84. DOI: 10.5433/1679-0367.2015v36n2p75.

18. Maciel ES, Sonati JG, Modeneze DM, Vasconcelos JS, Vilarta R. Consumo alimentar, estado nutricional e nível de atividade física em comunidade universitária brasileira. Rev Nutrição. 2012; 25(6):707-718.

19. Sousa TF, José HPM, Barbosa AR. Condutas negativas à saúde em estudantes universitários brasileiros. Ciência \& Saúde Colet. 2013; 28(12)3563-357. DOI: 10.1590/ S1413-81232013001200013.

20. López MJO, Nieto GP E, Aponte AF, Martínez M H. López GS, Martínez LMC. Evaluación Nutricional de una Población Universitaria. Rev Nut Hospitalar, 2006; 21(2):179-183.

21. Costa LCF, Vasconcelos FAG. Influência de fatores socioeconômicos, comportamentais e nutricionais na insatisfação com a imagem corporal de universitárias em Florianópolis, SC. Rev Bras Epidemiol. 2010; 13(4): 665-76.

22. Teo CRPA, Sá CA, Agnol PD, Welter S. Ambiente alimentar e vulnerabilidade de adolescentes universitários: Um estudo com foco no convívio familiar. Rev Bras Pesq Saúde. 2014; 16(1): 49-58.

23. Alves HJ.; Boong MCF. Comportamento Alimentar em moradia Estudantil: um espaço para promoção da saúde. Rev Saúde Pública. 2007; 41(2): 197-204.

24. Petribú MMV, Cabral PC, Arruda IK. Estado Nutricional, Consumo Alimentar e Risco Cardiovascular: um estudo em universitários. Rev Nutrição. 2009; 22(6): 837-846. DOI: $10.1590 / \mathrm{S} 1415-52732009000600005$.

25. Morales G, Guillen-Grima F, Muñoz S, Belmar C, Schifferli I, Muñoz A, Soto A. Factores de riesgo cardiovascular em universitarios de primer y tercer año. Rev Med Chile. 2017; 145(1): 299-308. 
26. Castro JBP, Vale RGS, Aguiar RS, Mattos RS. Perfil do estilo de vida de universitários de Educação Física da cidade do Rio de Janeiro. Rev Bras Ciência Movim 2017;25(2):73-83.

27. Mielke G I, Ramis TR, Habeyche EC, Oliz MM, Tessmer MGS, Azevedo MR, Hallal PC. Atividade Física e Fatores Associados em Universitários do Primeiro Ano da Universidade Federal de Pelotas. Rev Bras Ativ Física Saúde. 2010; 15(1): 57-64. DOI: 10.12820/rbafs.v.15n1p57-64.

28. Correa-Bautista JE et al. Comparison of Three Adiposity Indexes and Cutoff Values to Predict Metabolic Syndrome among University Students. Metab Synd Rel Dis. 2017; 20(20): 1-8. DOI: 10.1089/met.2017.0016

29. SBC. Sociedade Brasileira de Cardiologia. Atualização Da Diretriz Brasileira De Dislipidemias E Prevenção Da Aterosclerose - 2017. Rev Soc Bras Cardiol. 2017; 109(1): DOI: 10.5935/abc.20170121.

30. Lasatra CS, Hernández ET, Vicente VM, Castellanos MM, Sánchez MCG, Ramos MA, Vivajos LT, Roldán JLC. Pancreatitis Aguda por Hipertrigliceridemia. Ver Gastroenterol Hepatol. 2013; 36(4): 274-279. DOI:10.1016/j.gastrohep.2012.11.006.

31. Floody PD, Hormazábal MA, Navarrete FC. Análisis de los factores de riesgo cardiovascular en jóvenes universitarios según su estado nutricional. Nut Hospitalaria. 2015;32(4):1820-1824. DOI: 10.3305/ nh.2015.32.4.9502.

32. Carvalho CA, Fonseca PCA, Barbosa JB, Machado SP, Santos AM, Silva AAM. Associação entre Fatores de
Risco Cardiovascular e Indicadores Antropométricos de Obesidade em Universitários de São Luís, Maranhão, Brasil. Rev Ciência Saúde Col. 2015; 20(2): 479-490. DOI: $10.1590 / 1413-81232015202.02342014$.

33. Moreira OC, Costa AE, Oliveira CEP, Oliveira RAR, Brito ISS. Fatores de Risco Coronariano em Estudantes de uma Universidade Privada. Rev Bras Ciência Movimento. 2011; 19(2): 61-69.

34. Sandoval CEG, Burke YD, Mendizabal-Ruiz AP, Díaz EM, Morales JA. Prevalencia de obesidad y perfil lipídico alterado en jóvenes universitários. Nut Hospitalaria. 2014;29(2):315-321. DOI: 10.3305/nh.2014.29.2.7054.

35. Nahas MV. Estilo de Vida e o Conceito de Saúde Positiva: fundamentos da aptidão física relacionada à saúde. Florianópolis: Editora UFSC, 1989.

36. Pereira JC, Barreto SM, Passos VMA. Perfil de Risco Cardiovascular e Autoavaliação da Saúde no Brasil: estudo base populacional. Rev Panam Salud Pub, 2009; 25(6): 491-498.

37. Rovida TAS, Sumida DH, Santos AS, Moimaz SAS, Garbin CAS. Estresse e o estilo de vida dos acadêmicos ingressantes em um curso de graduação em Odontologia. Rev ABENO. 2015; 15(3):26-34.

38. Piegas LS, Avezum A, Pereira JCR, Neto JMR, Hoepfner C, Farran JA, Ramos FR, Timerman A, Esteves JP. Risk factors for myocardial infarction in Brazil. J Am Heart, 2003; 146(2): 331-338. DOI: 10.1016/S00028703(03)00181-9. 